\title{
Uso de aisladores de base en puentes de concreto simplemente apoyados
}

\section{Use of base isolators in simply supported concrete bridges}

\section{Leocadio Rico Pradilla}

Ingeniero civil, candidato a magister en ingeniería civil. Docente de la Universidad Industrial de Santander. Bucaramanga, Colombia.

Contacto:leocadio_rico@hotmail.com

\section{Gustavo Chio Cho}

Ingeniero Civil, doctor en Ingeniería de Caminos, Canales y Puertos. Docente de la Universidad Industrial de Santander. Bucaramanga, Colombia.

Contacto:gchioch@uis.edu.co

Fecha de recepción: 26 de noviembre de 2011

Clasificación del artículo: Reflexión

Fecha de aceptación: 28 de agosto de 2012

Financiamiento: Universidad Industrial de Santander

Palabras clave: aisladores de fricción, aisladores elastoméricos, aislamiento sísmico, análisis dinámico no lineal, demanda sísmica.

Key words: friction isolators, elastomeric isolators, seismic isolation, nonlinear dynamic analysis, seismic demand.

\section{RESUMEN}

En el presente artículo se presenta una comparación del comportamiento sísmico de puentes de concreto reforzado simplemente apoyados, usando aisladores de base de tres tipos, los aisladores de elastoméricos de alto amortiguamiento HDRB por sus siglas en inglés (High Damping Rubber Bearings), los aisladores elastoméricos con núcleo de plomo LRB (Lead Rubber Bearings) y el sistema de péndulo de fricción FPS (Friction Pen- dulum System). Se tomó como caso de estudio el viaducto la flora, definiéndose la geometría y su correspondiente modelamiento en SAP2000. Se definieron las propiedades mecánicas de los tres aisladores usando un modelo bilineal; para el modelo del viaducto se realizó un análisis dinámico no lineal de historias en el tiempo, considerando la no linealidad en los aisladores, usando los sismos de la falla Frontal y la falla BucaramangaSanta Marta, escalados a nivel de superficie. Se compararon los resultados del modelo sin aisla- 
miento sísmico versus los tres modelos con sistema de aislamiento sísmico, y se encontró que los diferentes sistemas de aislamiento sísmico reducen significativamente la demanda de los desplazamientos y fuerzas cortantes en las pilas del puente que le inducen los probables terremotos que puedan ocurrir.

\section{ABSTRACT}

The aim of this paper is to present a comparison of the seismic behavior of simply supported concrete bridges by using three types of base isolators, namely high damping rubber bearing (HDRB), lead rubber bearing (LRB) and friction pendulum system (FPS). "La Flora" viaduct is taken as case study; the geometry and the corresponding modeling of such viaduct are defined in sap2000. Additionally, the mechanical properties of three insulators are defined using a bilinear model. An analysis of histories in time was made for the model of the viaduct, considering the non-linearity in the insulators and using frontal fault earthquakes caused by the Frontal and Bucaramanga-Santa Marta fault; this is applied at a surface-level scale. The results from the model without seismic isolation are compared with the three systems of seismic isolation and the findings suggest that the different systems of seismic isolation significantly reduce both the demands of the displacements and the cutting forces in the piles of the bridge, which may induce probable earthquakes.

\section{INTRODUCCIÓN}

Los puentes son estructuras de vital importancia. Ellos actúan como un eslabón importante en la red de transporte terrestre y un daño serio en los mismos durante un evento sísmico, impedirá brindar la ayuda necesaria. La actividad sísmica de las últimas décadas ha puesto de manifiesto la vulnerabilidad de los puentes ante este fenómeno.

El control pasivo de estructuras es una de las estrategias que se han propuesto en los últimos años para controlar los desplazamientos y las fuerzas de inercia que se generan en los puentes durante un temblor. El concepto en el que se basa el control pasivo se orienta a la reducción de la demanda sísmica en la estructura y a mejorar su capacidad de disparar energía, más que en tratar de incrementar su capacidad de resistencia o de deformación. La adecuada aplicación de este criterio conduce a sistemas que se comportan en forma elástica durante grandes sismos; contraria-

mente al diseño tradicional, en el que se pretende mejorar la capacidad de disipar energía de la estructura mediante el daño en los elementos que la forman. Esto representa una importante ventaja, ya que al evitar el daño de los elementos de la estructura se consigue que permanezca en condiciones de funcionalidad después de ocurrido un siniestro [1].

En la figura 1(a) se ilustra el efecto de un sismo importante en una estructura típica de un puente, donde las grandes deformaciones generan agrietamientos importantes en las pilas, a las que se les exige una gran ductilidad para lograr disipar la energía del temblor sin que la estructura se derrumbe. En la figura 1(b) se presenta el caso de un puente con un sistema de aislamiento, con el cual se logran reducir las fuerzas de inercia en las pilas del puente. La disipación de energía inelástica que se demanda en un sismo extremo se realiza por medio de la deformación histerética de los apoyos, en lugar de hacerlo a través del daño en las pilas del puente. 


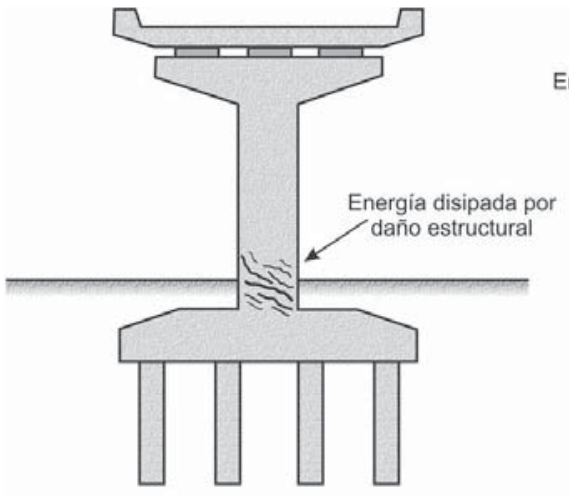

(a) Puente convencional

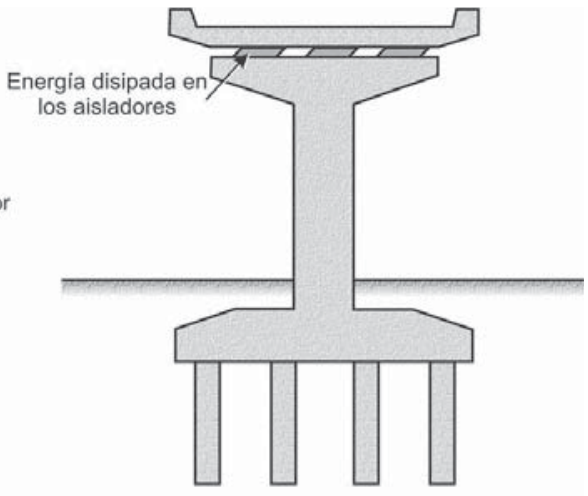

(a) Puente con aisladores

Figura 1. Disipación de energía en un puente convencional y en un puente con aislamiento de base.

Diferentes estudios realizados [2] - [7], han demostrado la efectividad de usar diferentes sistemas de aislamiento sísmico en puentes. En [8], se muestra un estado del arte detallado de estudios analíticos y experimentales sobre la efectividad de sistemas de aislamiento sísmico y su implementación actual en puentes.

\section{DESCRIPCIÓN Y MODELAMIENTO DEL VIADUCTO LA FLORA}

\subsection{Geometría}

El viaducto la flora es una estructura en concreto pretensado, construido con un sistema de pila y losa, con una longitud total de 298.3 metros.

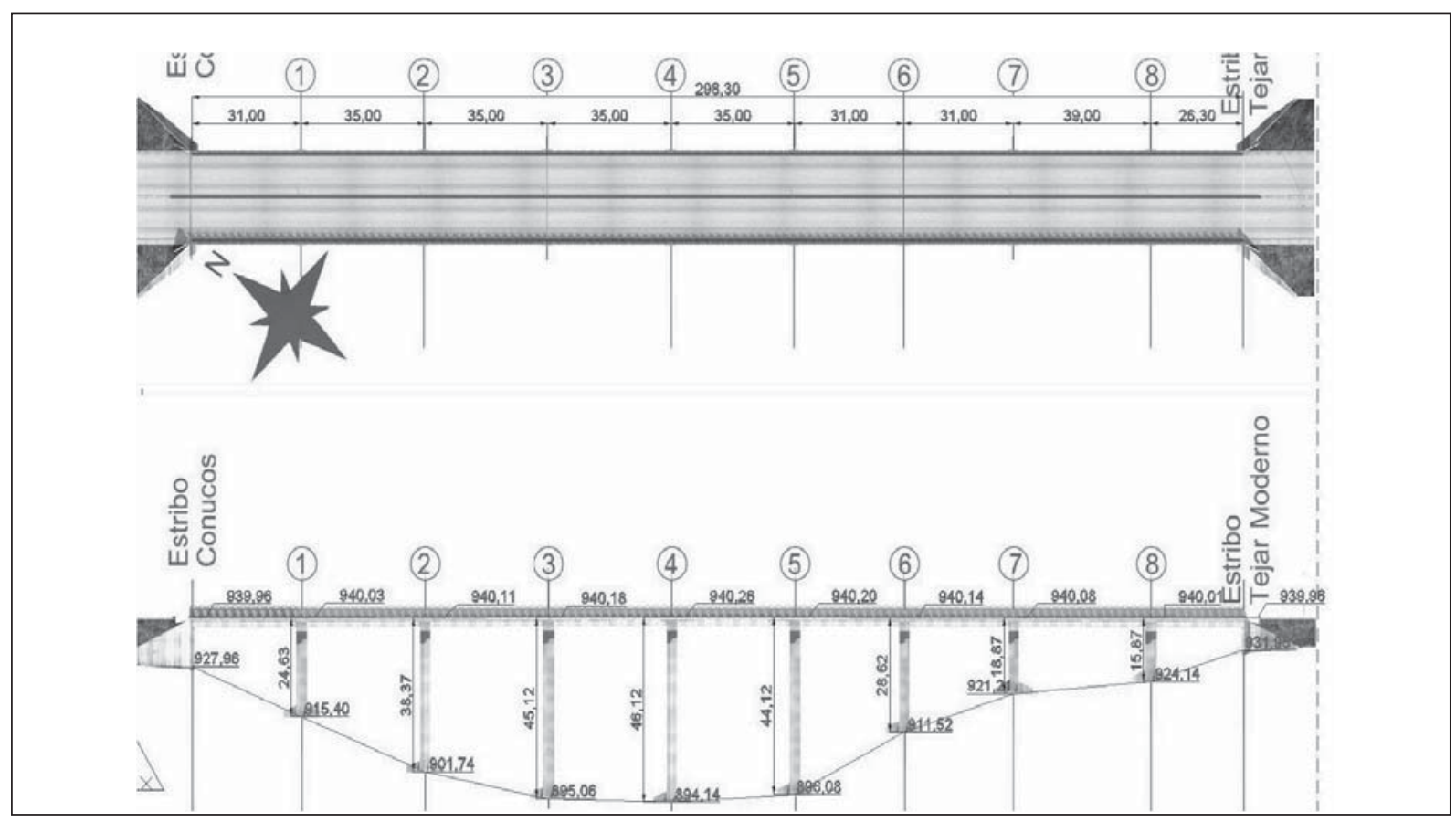

Figura 2. Planta-Perfil del viaducto La Flora. 


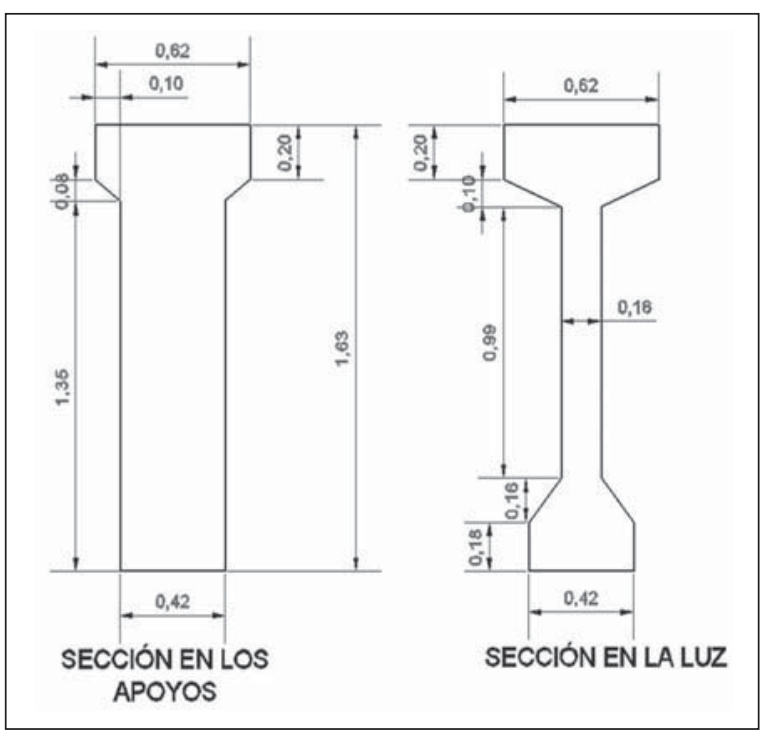

Figura 3. Sección transversal de las vigas.

Fuente: elaboración propia

Consta de dos estribos (estribo conucos al norte y estribo tejar moderno al sur) y ocho pilares. El tablero está divido en tres secciones, lo que da como resultado cuatro juntas ubicadas de la siguiente forma: dos en los estribos y las otras dos en los ejes 3 y 6 . La altura aproximada en el pilar más alto es de 46 metros que corresponde al eje 4 , y la luz más grande la encontramos entre los ejes 7 y 8 , de 39 metros. El puente tiene un ancho total de 25 metros y consta de tres carriles y sendero peatonal en cada sentido (ver figura 2).

Las vigas están conformadas por las secciones transversales que se muestran en la figura 3 y están construidas en concreto pretensado, las vigas se encuentran simplemente apoyadas en sus extremos (un extremo fijo y el otro móvil), en cada luz existen 10 vigas excepto en el tramo comprendido entre los ejes 7 y 8 , el cual tiene 11 vigas debido a la longitud de su luz de $39 \mathrm{~m}$.

Como ya se mencionó anteriormente, el puente consta de ocho pilares, los cuales varían su altura geométricamente según el perfil del terreno, en la tabla 1 se puede observar el dimensionamiento para cada uno de ellos. La cimentación de esta estructura se encuentra conformada por un par de zapatas aisladas en cada eje de cimentación con dimensión promedio de 7.5 y 7.0 metros y altura promedio de 2.5 metros. La estructura de apoyo se erige en cada eje con un par de pilas

Tabla 1. Dimensiones que varían para cada pilar del puente.

\begin{tabular}{|c|c|c|c|c|c|c|c|}
\hline Eje & Nivel Superior & Nivel Inferior & $H[\mathrm{~m}]$ & $\mathrm{B}[\mathrm{m}]$ & $\mathrm{L}[\mathrm{m}]$ & $\mathrm{H}[\mathrm{m}]$ & $\mathrm{h}^{\prime}[\mathrm{m}]$ \\
\hline 1 & 937.91 & 915.41 & 22.51 & 8.00 & 7.00 & 2.50 & 1.30 \\
\hline 2 & 937.99 & 901.74 & 36.25 & 9.50 & 7.50 & 2.75 & 1.50 \\
\hline 3 & 938.10 & 895.06 & 43.04 & 10.50 & 7.50 & 3.00 & 1.60 \\
\hline 4 & 938.14 & 894.14 & 44.00 & 10.50 & 7.50 & 3.00 & 1.60 \\
\hline 5 & 938.08 & 896.08 & 42.00 & 10.00 & 7.50 & 3.00 & 1.60 \\
\hline 6 & 938.02 & 911.52 & 26.50 & 8.00 & 7.00 & 2.50 & 1.30 \\
\hline 7 & 937.96 & 921.21 & 16.75 & 7.00 & 6.00 & 1.75 & 1.00 \\
\hline 8 & 937.89 & 924.14 & 13.75 & 7.00 & 6.00 & 1.75 & 1.00 \\
\hline
\end{tabular}

Fuente: elaboración propia 

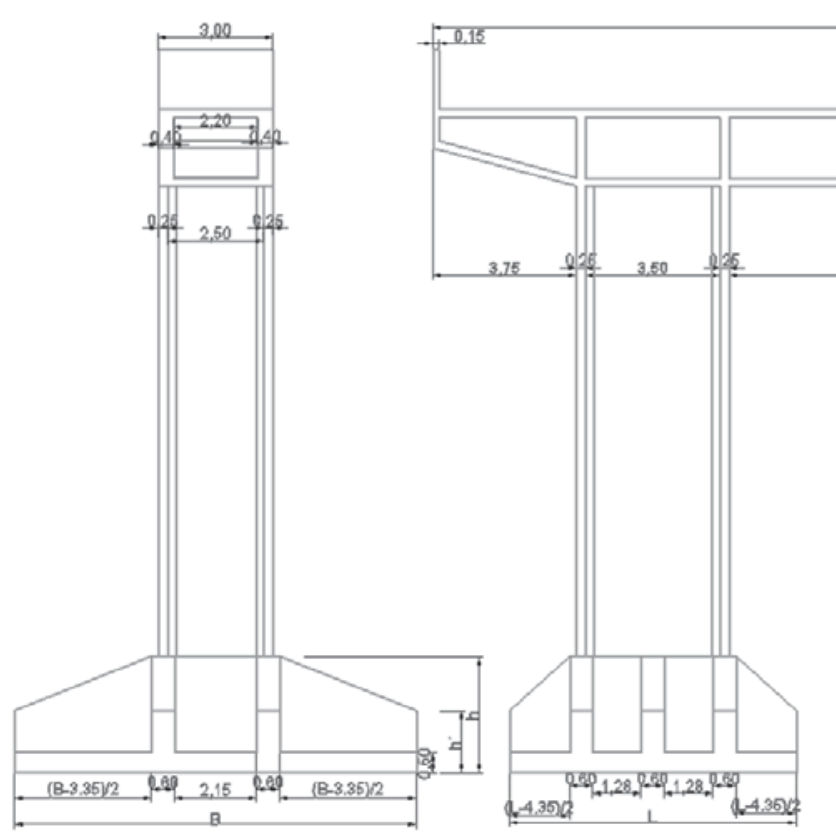

23,50
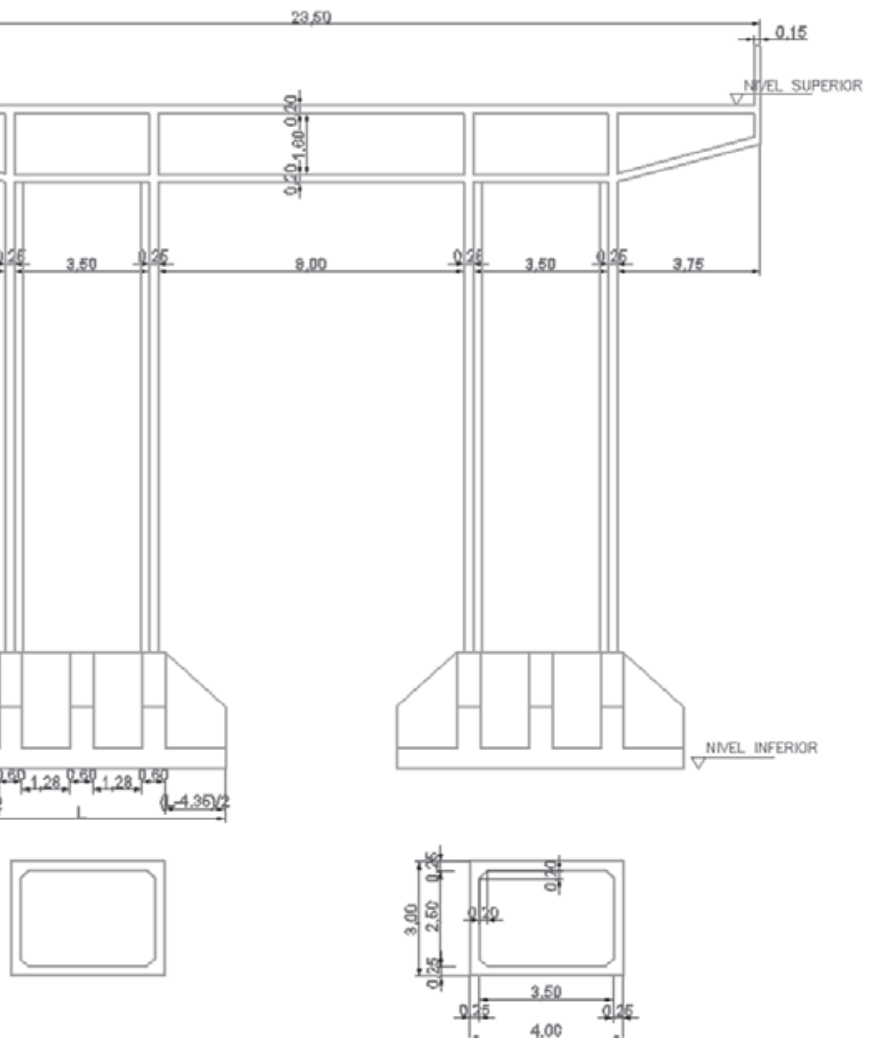

Figura 4. Sección tipo de los pilares.

Fuente: elaboración propia

conformadas por estructuras huecas rectangulares de 3 por 3 metros y espesor de 0.25 metros, con altura variable entre 15.87 y 46.12 metros y rematadas en una viga cabezal tipo cajón de 23.5 por 3 metros y 2 metros de altura, en la figura 4 se aprecian las proyecciones principales de un pilar tipo.

\subsection{Modelamiento del viaducto la flora en SAP2000}

El modelo del viaducto la Flora, se realizó en el software comercial SAP 2000 versión 14.2.2. Se definieron las secciones a utilizar, tanto para las vigas como para las pilas, se usaron elementos tipo frame. Dando como resultado el modelo tridimensional que se muestra en la figura 5.
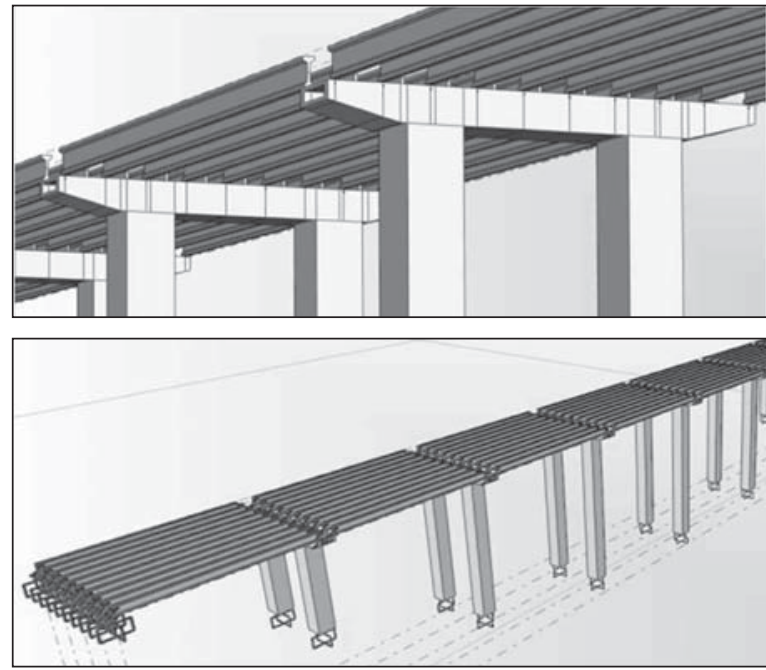

Figura 5. Detalle viga cabezal y proyección isométrica del viaducto la flora.

Fuente: SAP 2000 


\section{LOCALIZACIÓN Y TIPOS DE AISLADORES DE BASE}

\subsection{Localización de los aisladores}

Los aisladores de base estarán situados entre la interface de la viga cabezal y las vigas que dan soporte a la losa, en la figura 6 se muestra la posición donde se colocarían los aisladores. Para el presente trabajo, se realizarán los estudios pertinentes para tres tipos de aisladores, el HDRB, el LRB y el FPS.

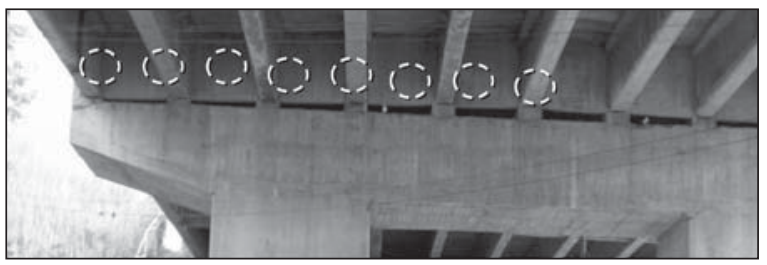

Figura 6. Detalle de la posición donde se instalarían los aisladores.

Fuente: elaboración propia

\subsection{Aisladores elastoméricos de alto amorti- guamiento (HDRB)}

Los aisladores de neopreno zunchado intercalan placas delgadas de acero en un bloque cúbico o cilíndrico de neopreno, una imagen y un esquema de un aislador HDRB se muestran en la figura 7. La rigidez vertical del HDRB aumenta considerablemente, manteniendo su flexibilidad lateral [9]. Los aisladores de alto amortiguamiento están hechos de un compuesto especial de goma, obtenido con la adición de carbono extra fino, aceite negro, o resinas, que permite alcanzar valores más altos de amortiguamiento. El espesor de las capas de caucho varía, normalmente, entre los $8 \mathrm{~mm}$ y 20 $\mathrm{mm}$, y el espesor de la capa de acero oscila entre $\operatorname{los} 2 \mathrm{~mm}$ y los $4 \mathrm{~mm}$.

Los valores de amortiguamiento varían entre el $10 \%$ y el 20\% [10], el análisis de estructuras aisladas con estos dispositivos se realiza como un sistema bilineal cuyas propiedades dependen de

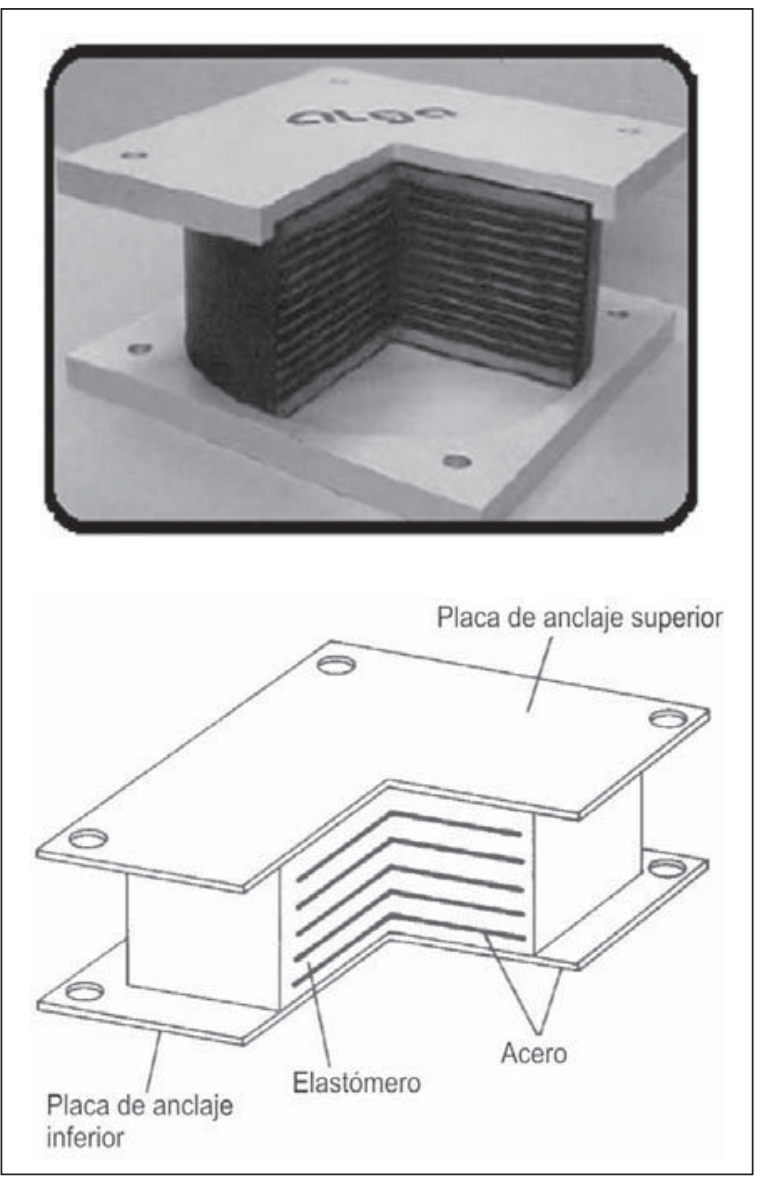

Figura 7. Aislador elastomérico de alto amortiguamiento (HDRB).

la razón de amortiguamiento efectivo y el módulo de deformación $(\mathrm{G})$ cuyos valores oscilan entre $0,4 \mathrm{MPa}$ y $1,4 \mathrm{MPa}$.

\subsection{Aisladores elastoméricos con núcleo de plomo (LRB)}

Estos aisladores son muy similares a los HDRB, con la diferencia que usan un núcleo de plomo que le proporciona una capacidad adicional de disipar energía y le brinda una rigidez inicial un poco más alta respecto a los anteriores, disminuyendo los desplazamientos para pequeños sismos y fuerzas de viento que actúen sobre la estructura [11], [12], (ver figura 8). 


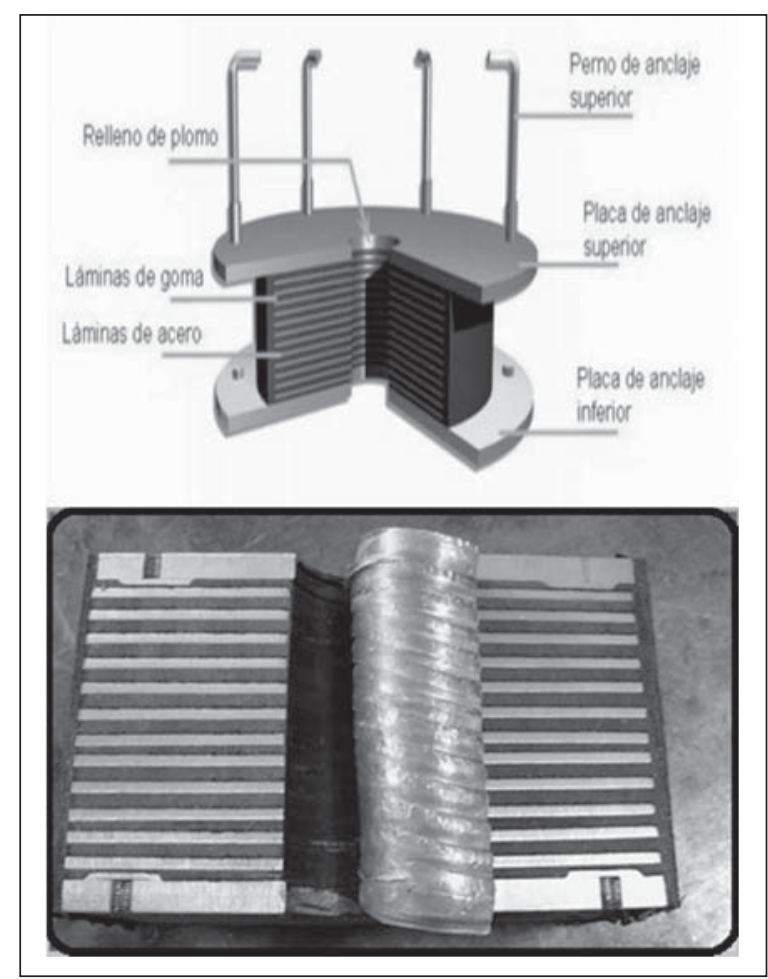

Figura 8. Aislador elastométrico con núcleo de plomo (LRB), (N-Z).

Estos aisladores inicialmente fueron desarrollados y usados en Nueva Zelanda, de ahí que algunos autores se refieran a ellos como sistemas N-Z. El comportamiento del aislador depende de la fuerza lateral que se impone, si la fuerza es muy pequeña será asumida por el núcleo de plomo y el sistema tendrá una alta rigidez, cuando la carga lateral se aumenta, el núcleo de plomo se deforma y empieza a fluir; el comportamiento histerético se genera con la energía disipada por el núcleo de plomo, como consecuencia la rigidez lateral del sistema disminuye. El amortiguamiento generalmente se encuentra entre el $15 \%$ y el $35 \%$ y se puede realizar el modelado de este aislador empleando la teoría bilineal. En su proceso de fabricación, es necesario que el hueco realizado en el caucho sea más pequeño que el diámetro del elemento de plomo, para que éste sea forzado a entrar. Esta característica garantiza la uniformidad del bloque y la consiguiente deformación por cortante de todo el volumen del núcleo de plomo. La plastificación del núcleo de plomo garantiza elevados valores de amortiguamiento, que originan, no sólo menores desplazamientos de la superestructura, sino también mayor rigidez lateral del tablero para niveles bajos de acciones horizontales. La tensión de cedencia a cortante del núcleo de plomo es de, aproximadamente, $10 \mathrm{MPa}$ sobrepasado este valor, la rigidez del dispositivo es la rigidez del caucho, lo que proporciona al dispositivo una rigidez bi-lineal. Este comportamiento es excelente, incluso a bajas temperaturas, pues el caucho presenta buenas características de resistencia a fatiga bajo estas condiciones. La rigidez inicial del aparato LRB es cerca de 10 veces superior a la rigidez poselástica, resultante de la cadencia del núcleo de plomo. Este tipo de sistema de aislamiento sísmico es la solución más utilizada en puentes, dada su simplicidad, comportamiento y bajo costo.

\subsection{Sistema de péndulo de fricción (FPS)}

El concepto de sistemas de deslizamiento se utiliza junto con la noción de una respuesta de tipo péndulo, por medio de un control deslizante articulado en una superficie esférica cóncava [13]; el sistema de péndulo de fricción está compuesto por un deslizador articulado sobre una superficie de acero inoxidable. La parte del apoyo articulado que está en contacto con la superficie esférica, está rodeada por una película de un material compuesto de baja fricción Teflón (Politetrafluoroetileno PTFE), ver figura 9.

La fuerza impuesta hace que se produzca desplazamiento en las direcciones horizontal y vertical, cuando esta fuerza desaparece se genera la fuerza restauradora, generando que el deslizador regrese al centro de la concavidad, el movimiento para cuando la fricción es igual o más grande que el componente de la carga vertical aplicada. La rigidez efectiva del aislador y el periodo de osci- 


\section{reflexión}

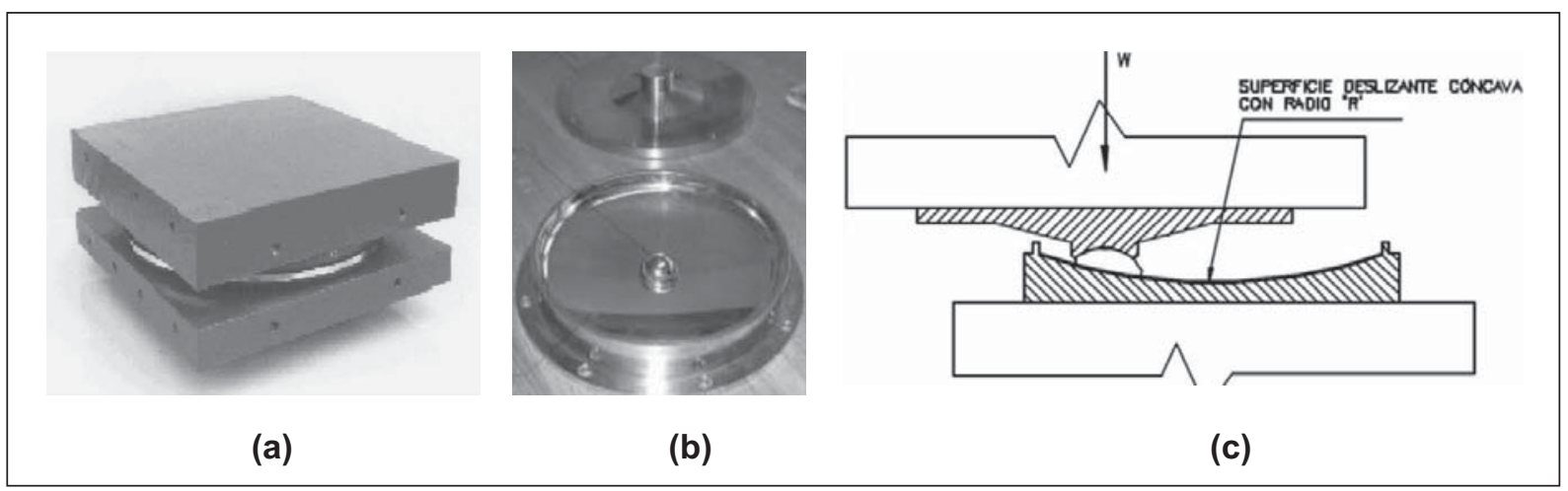

Figura 9. Sistema de péndulo de fricción (FPS). (a) Vista exterior. (b) Componentes internos. (c) Esquema de una sección transversal.

lación de la estructura están controlados por el radio de curvatura de la superficie cóncava, los movimientos de torsión de la estructura se reducen al mínimo, ya que el centro de la rigidez de los rodamientos de forma automática coincide con el centro de masa de la estructura de apoyo. Los rangos de amortiguamiento de este sistema se encuentran entre $10 \%$ y $40 \%$, por otra parte, los coeficientes de fricción están entre $3 \%$ y $20 \%$. El péndulo de fricción requiere un mantenimiento mínimo, ya que la superficie de teflón protege al deslizador de la corrosión, si el deslizamiento sólo se produce durante los sismos, se puede decir que el recubrimiento de teflón funcionará durante la vida útil del diseño; incluso se han desarrollado dispositivos con capacidad de resistir fuerzas a tensión. Recientemente, se han desarrollado péndulos de fricción con dos y tres superficies cóncavas, produciendo el mismo movimiento horizontal y disminuyendo el tamaño del aislador.

\section{CARACTERÍSTICAS MECÁNICAS Y MODELAMIENTO DE LOS AISLADORES DE BASE}

Teóricamente, la no linealidad de una estructura aislada de su base proviene de dos fuentes: las deformaciones inelásticas en el sistema de resistencia sísmica de la estructura y el sistema de aislación: sin embargo, considerando que el sistema de resistencia sísmica es más rígido que el sistema de aislación, se espera que las deformaciones inelásticas se encuentren localizadas en los aisladores y la estructura sobre estos presente un comportamiento lineal.

Para hacer un uso seguro de los aisladores, las propiedades mecánicas de diferentes tipos de aisladores han sido investigadas intensamente. El modelo bilineal ha sido ampliamente aceptado para la investigación y el diseño, esto se debe a que caracteriza las propiedades mecánicas de los aisladores adecuadamente pero también a que es válido tanto para aisladores elastométricos como para aisladores de fricción.

\subsection{Parámetros del modelo bilineal}

El modelo bilineal usado para expresar la relación entre la fuerza cortante y el desplazamiento lateral, puede definirse por tres parámetros básicos: Rigidez Elástica, $k_{e}$, Rigidez Post-fluencia, $k_{p}$, Fuerza Característica, Q. La fuerza característica $Q$ se emplea a menudo para estimar la estabilidad del comportamiento histerético, cuando el aislador experimenta muchos ciclos de carga. Estos tres parámetros reflejan adecuadamente las características mecánicas de los aisladores de 


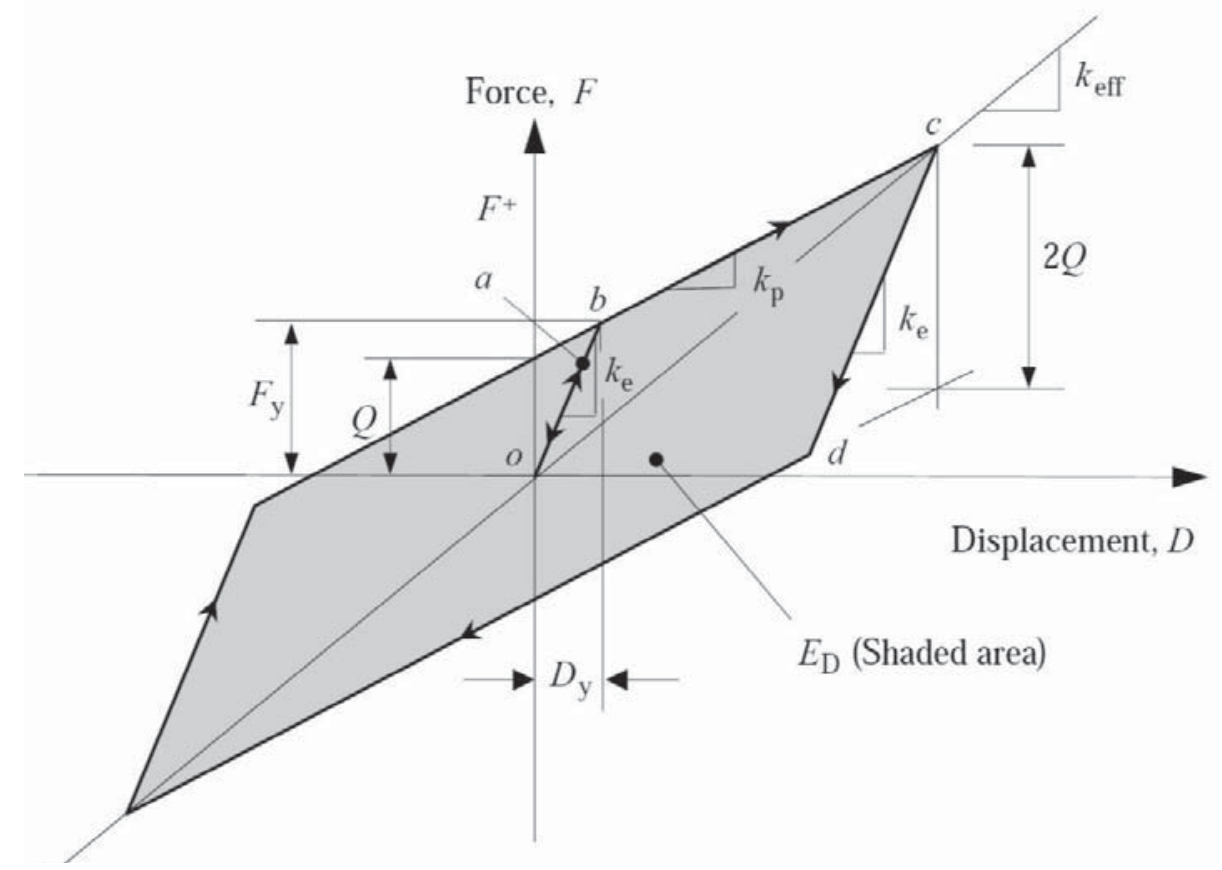

Figura 10. Modelo bilineal del aislador.

Fuente: elaboración propia

una forma simple y suministran una estimación satisfactoria del comportamiento no lineal de un aislador. La figura 10 muestra un modelo bilineal idealizado,

La rigidez efectiva $k_{\text {eff }}$, en la región de posfluencia puede ser expresada en términos de la rigidez posfluencia $k_{p}$ y la fuerza característica $Q$ con el correspondiente desplazamiento $D$ lateral. De esta manera tenemos la ecuación (1):

$$
k_{e f f}=k_{p}+Q / D
$$

El desplazamiento de fluencia $D_{y}$, también se deriva de $k_{e}, k_{p}, Q$ ecuación (2):

$$
D_{y}=\frac{Q}{k_{e}-k_{p}}
$$

La fuerza de fluencia $F_{y}$, en el desplazamiento de fluencia $D_{y}$ se determina a través de la ecuación (3):

$$
F_{y}=Q+k_{p} D_{y}
$$

El amortiguamiento efectivo $\beta_{\text {eff }}$, se define como en la ecuación (4):

$$
\beta_{e f f}=\frac{E_{D}}{2 \pi k_{e f f} D^{2}}
$$

Donde $D_{E}$ es considerada como el área del ciclo de histéresis y corresponde a la energía disipada por ciclo, limitada por el desplazamiento lateral $-D$ y $+D$ en cada ciclo por lo tanto $D_{E}=4 Q(D-$ $\left.D_{y}\right)$ Ec. (5).

$$
\beta_{e f f}=\frac{4 Q\left(D-D_{y}\right)}{2 \pi k_{e f f} D^{2}}=\frac{2 Q\left(D-D_{y}\right)}{\pi k_{e f f} D^{2}}
$$

En el diseño estructural, tanto la rigidez efectiva como el amortiguamiento efectivo, se determinan a partir del desplazamiento de diseño (DD) y el desplazamiento máximo (DM), los cuales se definen para un periodo fundamental del sistema. 


\subsection{Modelo bilineal del aislador con núcleo de plomo}

La fuerza característica $Q$ de los aisladores con núcleo de plomo es controlada principalmente por la fuerza cortante del núcleo de plomo. El cortante de fluencia ocurre en el núcleo de plomo a bajos niveles de esfuerzo cortante. Sin embargo, el comportamiento histerético del aislador es bastante estable, inclusive cuando éste es sometido a muchos ciclos de carga. La siguiente ecuación muestra la relación que existe entre la fuerza característica $Q$ y el producto del esfuerzo de fluencia $f_{y_{1}}$ del plomo por el área de plomo $A_{1}$, como se puede ver en la ecuación (6), este dato es característico para el aislador con núcleo de plomo:

$$
Q=A_{1} f_{y 1}
$$

La rigidez post-fluencia $k_{p}$, se -define a continuación en la ecuación (7) [14].

$$
k_{p}=\frac{A_{b} G f_{L}}{t}
$$

Donde, $A_{b}$ es el área de caucho, $t$ es el grosor total del caucho, $f_{L}=1,5$ y $\mathrm{G}$ es el módulo de cortante tangente del caucho (se determina a través de pruebas dinámicas de cortante). La rigidez elástica $k_{e}$ no es fácil de calcular, pero a través de la siguiente ecuación empírica se puede obtener un valor aproximado, la rigidez elástica se define como un múltiplo de la rigidez posfluencia, ecuación (8), esto se puede escribir como:

$$
6.5 k_{p} \leq k_{e} \leq 10 k_{p}
$$

En base a esta condición se puede conocer el desplazamiento de fluencia $D_{y}$ sustituyendo los valores encontrados y asumiendo que $k_{e}=k k_{p}$. Se obtiene la ecuación (9) [20]:

$$
D_{y}=\frac{Q}{k_{e}-k_{p}} \approx \frac{Q}{\kappa k_{p}-k_{p}}=\frac{Q}{(\kappa-1) k_{p}} \text { (9) }
$$

Sustituyendo los datos encontrados hasta el momento, se puede encontrar la ecuación (10); el amortiguamiento efectivo para un aislador con núcleo de plomo.

$$
\beta_{e f f}=\frac{2 Q\left(D-D_{y}\right)}{\pi k_{e f f} D^{2}}=\frac{2 Q\left[(\kappa-1) k_{p} D-Q\right]}{\pi(\kappa-1) k_{p}\left(k_{p} D+Q\right) D}(10)
$$

En la tabla 2, se muestran todas y cada una de las características requeridas para el modelamiento del aislador LRB, para determinar la rigidez ver-

Tabla 2. Características mecánicas del aislador LRB

\begin{tabular}{|l|r|r|l|r|r|}
\hline \multicolumn{7}{|c|}{ AISLADOR LRB } & 200 & $\mathrm{Mm}$ \\
\hline Ancho & 400 & $\mathrm{Mm}$ & $\mathrm{t}$ grosor total del caucho & $1.026,99$ & $\mathrm{~N} / \mathrm{mm}$ \\
\hline Largo & 400 & $\mathrm{Mm}$ & $\mathrm{K}_{\mathrm{p}}$ rigidez post-fluencia & $10.269,86$ & $\mathrm{~N} / \mathrm{mm}$ \\
\hline Alto & 250 & $\mathrm{Mm}$ & $\mathrm{K}_{\mathrm{e}}$ rigidez elastica & 200 & $\mathrm{Mm}$ \\
\hline Espesor lámina de caucho & 8 & $\mathrm{Mm}$ & $\mathrm{D}$ desplazamiento de diseño & $1.419,68$ & $\mathrm{~N} / \mathrm{mm}$ \\
\hline espesor lámina de acero & 2 & $\mathrm{Mm}$ & $\mathrm{K}_{\text {eff }}$ rigidez efectiva & 8,50 & $\mathrm{Mm}$ \\
\hline Diámetro Núcleo de plomo & 100 & $\mathrm{Mm}$ & $\mathrm{D}_{\mathrm{y}}$ desplazamiento de fluencia & $87.266,46$ & $\mathrm{~N}$ \\
\hline $\mathrm{f}_{\mathrm{y} 1}$ esfuerzo de fluencia del plomo & 10 & $\mathrm{Mpa}$ & $\mathrm{F}_{\mathrm{y}}$ fuerza de fluencia & $16,86 \%$ & \\
\hline G módulo de cortante del caucho & 0,9 & $\mathrm{Mpa}$ & $\square_{\text {eff }}$ amortiguamiento efectivo & $709.842,35$ & $\mathrm{~N} / \mathrm{mm}$ \\
\hline $\mathrm{A}_{1}$ área de plomo & $7.853,98$ & $\mathrm{~mm}^{2}$ & Rigidez vertical del aislador & 0,100 & \\
\hline Q fuerza característica & $78.539,82$ & $\mathrm{~N}$ & Relación $\left(\mathrm{k}_{\mathrm{p}} / \mathrm{k}_{\mathrm{e}}\right.$ ) & & \\
\hline $\mathrm{A}_{\mathrm{b}}$ área de caucho & $152.146,02$ & $\mathrm{~mm}^{2}$ & & & \\
\hline
\end{tabular}

Fuente: elaboración propia 
tical del aislador se tomará como 500 veces la rigidez efectiva horizontal.

\subsection{Modelo bilineal del aislador de alto amortiguamiento}

Para generar un modelo bilineal para un aislador elastométrico de alto amortiguamiento, se emplean parámetros que son normalmente derivados del modulo de cortante $G$ y el amortiguamiento efectivo $\beta_{\text {eff }}$. El modulo de cortante tangente $G$, es determinado con precisión de una prueba dinámica de cortante y el amortiguamiento efectivo, determinado de las pruebas a los prototipos de aisladores, puede variar entre $10 \%$ y $20 \%$ del amortiguamiento critico, [10]. La ecuación para calcular la rigidez posfluencia $k_{p}$ para este tipo de aisladores es:

$$
k_{p}=\frac{A_{b} G}{t}
$$

Donde, $A_{b}$ es el área de caucho, $Q$ es el grosor total del caucho, $\mathrm{G}$ es el módulo de cortante tangente del caucho. La fuerza característica $Q$ puede ser evaluada por la ecuación (12):

$$
Q=\frac{\pi \beta_{e f f} k_{p} D_{D}^{2}}{\left(2-\pi \beta_{e f f}\right) D_{D}-2 D_{y}}
$$

Una estimación aproximada de $d_{y}$ puede ser expresada en términos del espesor total de la goma $t, D_{y}=\lambda t$, donde el coeficiente $\lambda$ varía entre 0,05 y 0,1 [14].

Una vez son conocidas la rigidez posfluencia $k_{p}$, el desplazamiento de fluencia $D_{y}$ y la fuerza característica $Q$, la fuerza de fluencia es fácilmente determinada por la ecuación (13).

$$
F_{y}=Q+k_{p} D_{y}
$$

Luego la rigidez elástica de los aisladores de alto amortiguamiento se convierte en, la ecuación (14).

$$
\begin{gathered}
k_{e}=\frac{F_{y}}{D_{y}}=k_{p}+\frac{Q}{D_{y}}=k_{p} \\
\left\{1+\frac{\pi \beta_{\text {eff }} k_{p} D_{D}^{L}}{\lambda t\left[\left(2-\pi \beta_{e f f}\right) D_{D}-2 \lambda t\right]}\right\}
\end{gathered}
$$

Finalmente, si se sustituye $D_{y}=\lambda t$, la rigidez efectiva, hasta lograrse el desplazamiento de diseño estará dada por la ecuación (15):

$$
k_{e f f}=\frac{2 Q\left(D_{D}-\lambda t\right)}{\pi \beta_{e f f} D_{D}^{2}}
$$

En la tabla 3, se muestran cada una de las características requeridas para el modelamiento del aislador HDRB, para determinar la rigidez vertical del aislador se tomará como 500 veces la rigidez efectiva horizontal.

\subsection{Modelo bilineal del sistema de péndulo de fricción}

La fuerza característica $Q$ de un péndulo de fricción, es expresada en la ecuación (16) como:

$$
Q=\mu_{S} P_{C}
$$

Donde, $P_{\mathrm{C}}$ es la fuerza axial aplicada sobre el aislador, la cual está compuesta por la carga gravitacional $P_{\mathrm{g}}$ y el efecto de la aceleración vertical del terreno. Despreciando los efectos de la aceleración vertical la fuerza axial $P_{\mathrm{C}}=P_{\mathrm{g}}$.

$\mu_{\mathrm{S}}=$ Coeficiente de fricción que está relacionado a la velocidad de deslizamiento y se calcula empleando la ecuación (17) [15].

$$
\mu_{S}=f_{\max }-\left(f_{\max }-f_{\min }\right) e^{-\xi|\dot{D}|}
$$

Donde, $f_{\max } y f_{\min }$ son coeficientes de fricción calculados a alta y baja velocidad respectivamente, D representa la velocidad de movimiento del aislador, $\xi$ es la inversa de la velocidad de deslizamiento característica, este parámetro controla 


\section{reflexión}

Tabla 3. Características mecánicas del aislador HDRB

\begin{tabular}{|c|c|c|c|c|c|}
\hline \multicolumn{6}{|l|}{ AISLADOR HDRB } \\
\hline Ancho & 400 & $\mathrm{Mm}$ & $D_{D}$ desplazamiento de diseño & 200 & $\mathrm{Mm}$ \\
\hline Largo & 400 & $\mathrm{Mm}$ & $\square$ parámetro que varia entre 0,05 y 0,1 & 0,07 & \\
\hline Alto & 250 & $\mathrm{Mm}$ & $D_{y}$ desplazamiento de fluencia & 17,50 & $\mathrm{Mm}$ \\
\hline Espesor lámina de caucho & 8 & $\mathrm{Mm}$ & Q fuerza característica & $40.100,67$ & $\mathrm{~N}$ \\
\hline G módulo de cortante del caucho & 0,9 & Mpa & $F_{y}$ fuerza de fluencia & $50.180,67$ & $\mathrm{~N}$ \\
\hline $\mathrm{A}_{\mathrm{b}}$ área de caucho & 160 & $\mathrm{~mm}^{2}$ & $\mathrm{~K}_{\mathrm{e}}$ rigidez elástica & $2.867,47$ & $\mathrm{~N} / \mathrm{mm}$ \\
\hline t grosor total del caucho & 250 & $\mathrm{Mm}$ & $\mathrm{K}_{\text {eff }}$ rigidez efectiva & 776,50 & $\mathrm{~N} / \mathrm{mm}$ \\
\hline $\mathrm{K}_{\mathrm{p}}$ rigidez post-fluencia & 576,00 & $\mathrm{~N} / \mathrm{mm}$ & Rigidez vertical del aislador & $388.251,66$ & $\mathrm{~N} / \mathrm{mm}$ \\
\hline$\square_{\text {eff }}$ amortiguamiento efectivo & $15,0 \%$ & & Relación $\left(k_{p} / k_{e}\right)$ & 0,201 & \\
\hline
\end{tabular}

Fuente: elaboración propia

la transición de $f_{\max }$ a $f_{\min }$ la cual es calculada en base a experimentos, sin embargo, algunos autores sugieren un valor aproximado de $100 \mathrm{~s} / \mathrm{m}$ [15]. La rigidez posfluencia $k_{p}$ para los aisladores que utilizan péndulo de fricción, se calcula a través de la ecuación (18).

$$
k_{p}=\frac{P_{c}}{R}
$$

Donde, $\mathrm{R}$ representa el radio de curvatura de la superficie deslizante, los resultados de los experimentos indican que la rigidez elástica $k_{e}$ basados en muchos experimentos que se han realizado alrededor de este sistema, se ha determinado que es, normalmente, al menos 100 veces más grande que la rigidez posfluencia $k_{p}$. De acuerdo con esto, se define al desplazamiento de fluencia en la ecuación (19) como:

$$
D_{y}=\frac{Q}{k_{e}-k_{p}} \approx \frac{Q}{100 k_{p}}=\frac{\mu_{S} P_{C}}{100\left(P_{c} / R\right)}=\frac{\mu_{S} R}{100}
$$

Esta ecuación indica que el desplazamiento de fluencia $D_{y}$ es un valor muy pequeño y que se puede aproximar a $0,00254 \mathrm{~m}$. La ecuación (20) de la rigidez efectiva para el sistema de péndulo de fricción se puede escribir como:

$$
k_{e f f}=k_{p}+\frac{Q}{D}=P_{c}\left(\frac{1}{R}+\frac{\mu_{s}}{D_{D}}\right)
$$

Como el desplazamiento de fluencia $D_{y}$ es tan pequeño, comparándolo con el desplazamiento de diseño $D_{D}$, se puede ignorar para desarrollar la ecuación (21) del área del ciclo de histéresis.

$$
E_{D}=4 Q\left(D_{D}-D_{y}\right) \approx 4 Q D_{D}=4 \mu_{s} P_{c} D
$$

Finalmente, se puede obtener la ecuación (22) el amortiguamiento efectivo correspondiente a este dispositivo:

$$
\begin{gathered}
\beta_{e f f}=\frac{E_{D}}{2 \pi k_{e f f} D^{2}}=\frac{4 \mu_{s} P_{c} D_{D}}{2 \pi\left(1 / R+\mu_{s} / D_{D}\right) D_{D}^{2}} \\
=\frac{2 \mu_{S}}{\pi\left(D_{D} / R+\mu_{s}\right)}
\end{gathered}
$$

En la tabla 4, se muestran cada una de las características requeridas para el modelamiento del aislador FPS, para determinar la rigidez vertical del aislador se tomará como 500 veces la rigidez efectiva horizontal. 
Tabla 4. Características mecánicas del aislador FPS

\begin{tabular}{|l|r|r|l|r|r|}
\hline AISLADOR FPS & 400 & $\mathrm{Mm}$ & Kp rigidez post-fluencia & 350,04 & $\mathrm{~N} / \mathrm{mm}$ \\
\hline Diámetro del disco deslizante & 1.5 & $\mathrm{Mm}$ & Ke rigidez elástica & $35.004,00$ & $\mathrm{~N} / \mathrm{mm}$ \\
\hline Radio curvatura & 525.06 & $\mathrm{~N}$ & Dy desplazamiento de fluencia & 0,90 & $\mathrm{~mm}$ \\
\hline PC fuerza axial sobre el aislador & 0,06 & & DD desplazamiento de diseño & 200 & $\mathrm{~mm}$ \\
\hline fmáx & 0,04 & & Keff rigidez efectiva & 507,56 & $\mathrm{~N} / \mathrm{mm}$ \\
\hline fmín & 100 & $\mathrm{~s} / \mathrm{m}$ & Fy fuerza de fluencia & $31.818,15$ & $\mathrm{~N}$ \\
\hline X & 0,10 & $\mathrm{~m} / \mathrm{s}$ & $\square$ eff amortiguamiento efectivo & $19,76 \%$ & \\
\hline Ď & 0,060 & & Rigidez vertical del aislador & $253.777,81$ & $\mathrm{~N} / \mathrm{mm}$ \\
\hline$\square$ S & $31.503,12$ & $\mathrm{~N}$ & & &
\end{tabular}

Fuente: elaboración propia

\section{DEFINICIÓN DE LA ACCIÓN SÍSMICA}

\subsection{Definición de los acelerogramas}

El estudio de zonificación sismogeotécnica indicativa del área metropolitana de Bucaramanga presentado por INGEOMINAS [16], indica que la amenaza para la ciudad de Bucaramanga está controlada por la posible ocurrencia de un sismo a lo largo de las fallas Bucaramanga-Santa Marta,
Suárez, Salinas y la Frontal de los Llanos Orientales. Sin embargo, este mismo estudio propone tomar como escenarios de amenaza la Falla Bucaramanga-Santa Marta, y la Falla Frontal de los Llanos Orientales. Lo anterior se debe a que los posibles efectos generados por un sismo en las fallas Suárez y Salinas estarían cubiertos por los escenarios escogidos. Además, debido a que estos registros generados por INGEOMINAS muestran los datos de aceleración producida por el sismo
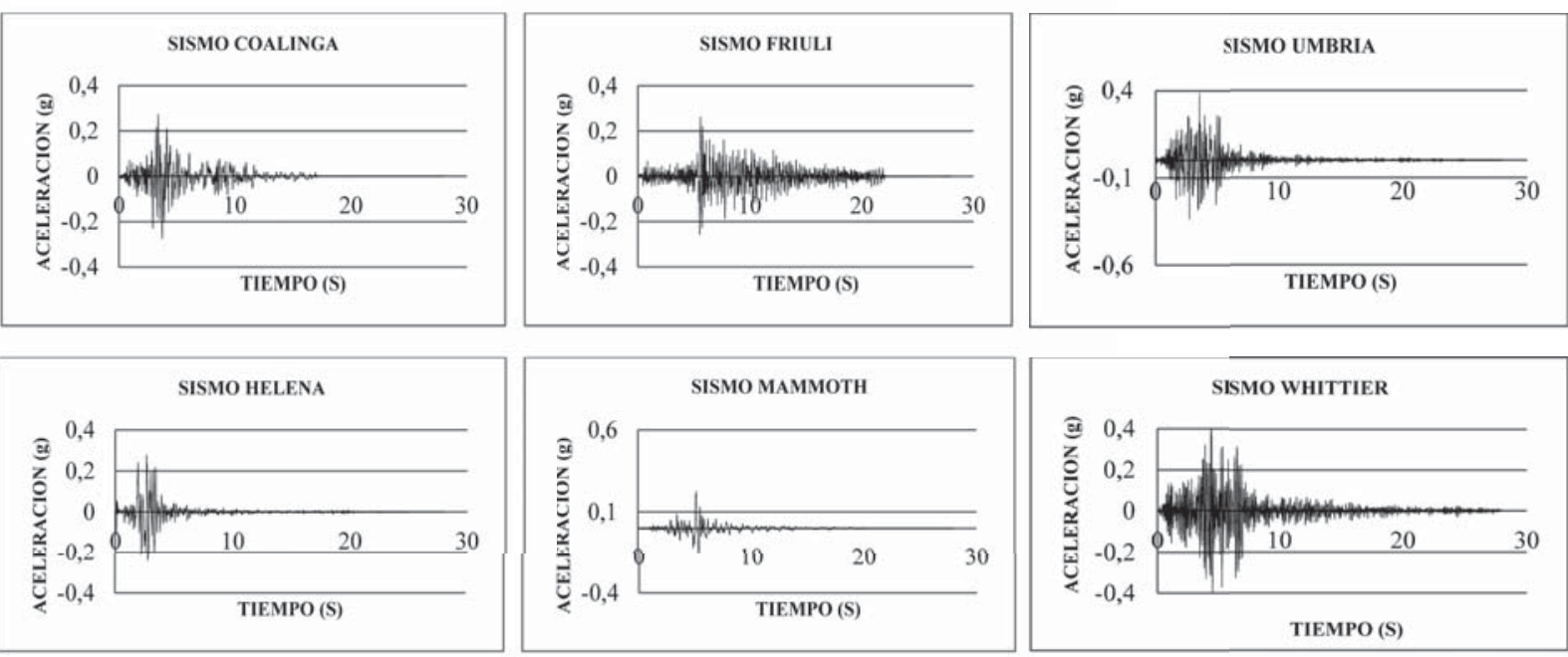

Figura 11. Acelerogramas compatibles con escenario sísmico falla Bucaramanga-Santa Marta para la zona 2.

Fuente: elaboración propia 


\section{reflexión}
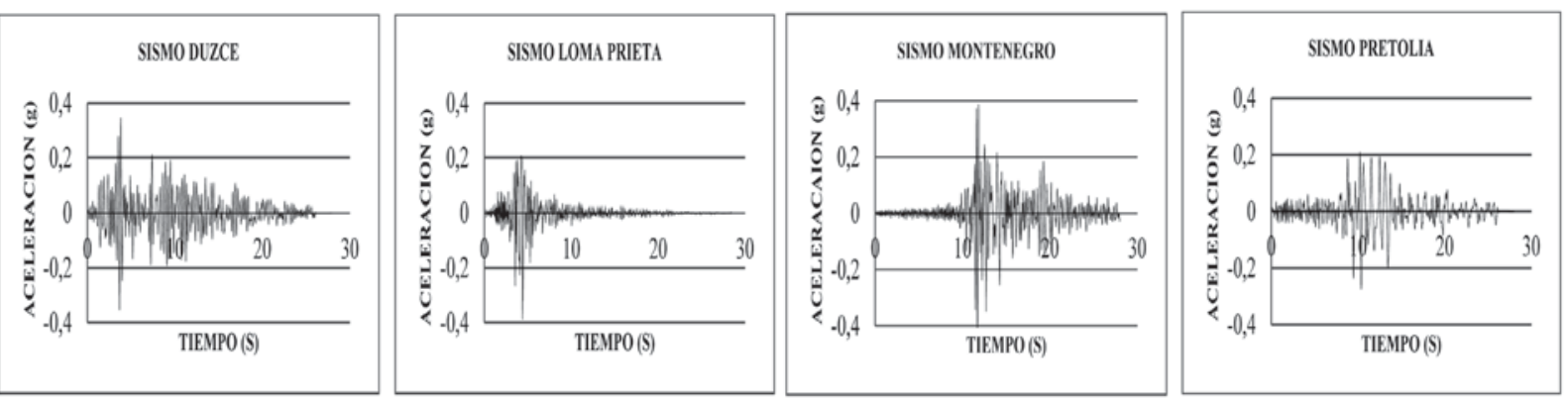

Figura 12. Acelerogramas compatibles con escenario sísmico falla Frontal para la zona 2.

Fuente: elaboración propia

directamente en la roca $y$, como en la ciudad de Bucaramanga generalmente las estructuras no están cimentadas sobre roca sólida, es necesario, para un mejor análisis, obtener los registros de los acelerogramas en la superficie del terreno [17]. Realizaron el filtro de estas señales teniendo en cuenta la zonificación sismogeotécnica hecha por INGEOMINAS, la cual depende de las características del suelo y la respuesta dinámica de cada zona de la región generando estos acelerogramas en superficie, como se muestran en la figura $11 \mathrm{y}$ en la figura 12.

\section{ANÁLISIS Y COMPARACIÓN DE RESULTADOS}

\subsection{Periodos y modos de vibración}

En la tabla 5 se muestran los periodos y frecuencias para los primeros ocho modos de vibración del puente sin sistema de aislamiento y con los tres sistemas de aisladores usados.

Para los cuatro modelos del puente, el primer modo de vibración está en dirección longitudinal. Es de aclarar también que, para el modelo sin ais-

Tabla 5. Comparación de los periodos para los modelos del viaducto sin sistema de aislamiento, con HDRB, con FPS y con LRB

\begin{tabular}{|c|c|c|c|c|c|c|c|c|}
\cline { 2 - 10 } \multicolumn{1}{c|}{} & \multicolumn{2}{c|}{ SIN AISLADOR } & \multicolumn{2}{c|}{ HDRB } & \multicolumn{3}{c|}{ FPS } \\
\hline $\begin{array}{c}\text { Modo } \\
\text { No. }\end{array}$ & $\begin{array}{c}\text { Periodo } \\
\text { [seg] }\end{array}$ & $\begin{array}{c}\text { Frecuencia } \\
\text { [ciclos/seg] }\end{array}$ & $\begin{array}{c}\text { Periodo } \\
\text { [seg] }\end{array}$ & $\begin{array}{c}\text { Frecuencia } \\
\text { [ciclos/seg] }\end{array}$ & $\begin{array}{c}\text { Periodo } \\
\text { [seg] }\end{array}$ & $\begin{array}{c}\text { Frecuencia } \\
\text { [ciclos/seg] }\end{array}$ & $\begin{array}{c}\text { Periodo } \\
\text { [seg] }\end{array}$ & $\begin{array}{c}\text { Frecuencia } \\
\text { [ciclos/seg] }\end{array}$ \\
\hline 1 & 2.91 & 0.34 & 2.32 & 0.43 & 2.61 & 0.38 & 2.02 & 0.49 \\
\hline 2 & 2.80 & 0.36 & 2.21 & 0.45 & 2.51 & 0.40 & 1.91 & 0.52 \\
\hline 3 & 2.56 & 0.39 & 2.03 & 0.49 & 2.37 & 0.42 & 1.66 & 0.60 \\
\hline 4 & 2.12 & 0.47 & 1.96 & 0.51 & 2.30 & 0.44 & 1.59 & 0.63 \\
\hline 5 & 1.19 & 0.84 & 1.83 & 0.55 & 2.19 & 0.46 & 1.43 & 0.70 \\
\hline 6 & 0.96 & 1.04 & 1.78 & 0.56 & 2.15 & 0.47 & 1.39 & 0.72 \\
\hline 7 & 0.62 & 1.62 & 1.71 & 0.59 & 2.09 & 0.48 & 1.30 & 0.77 \\
\hline 8 & 0.40 & 2.51 & 1.70 & 0.59 & 2.09 & 0.48 & 1.28 & 0.78 \\
\hline
\end{tabular}

Fuente: elaboración propia 
DESPLAZAMIENTO EN DIRECCIÓN LONGITUDINAL DEL PUENTE (EJE X) DE LA PILA 3 PARA EL SISMO DE DUZCE [mm]

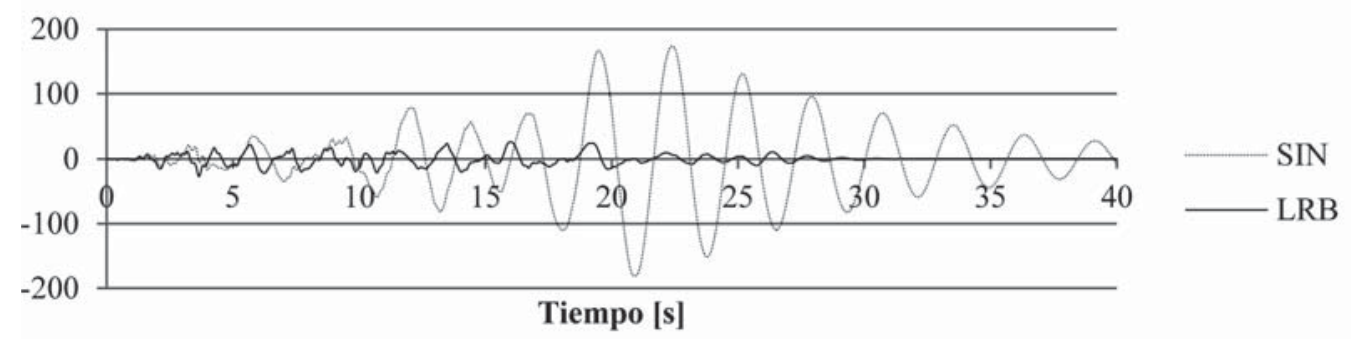

Figura 13. Desplazamiento de la pila 3 en dirección longitudinal del puente para el sismo de Duzce, sin aislamiento versus el aislamiento LRB.

Fuente: elaboración propia

ladores, los primeros ocho modos de vibración ocurren en dirección longitudinal, mientras que para los modelos con aisladores, el primer y quinto modo de vibración están en dirección longitudinal, y el segundo y sexto modo de vibración ocurren en dirección transversal.

\subsection{Desplazamientos y fuerzas cortantes en la base}

Como ejemplo particular, en la figura 13 se muestra la comparación de los desplazamientos en la pila 3 en dirección longitudinal para el puente sin aislamiento sísmico versus el aislamiento LRB para el sismo de Duzce. De igual manera, se compara la fuerza cortante en la pila 7, para el sismo de Pretolia, para el puente sin aislamiento sísmico versus el aislamiento HDRB, ver figura 14.

En la tabla 6 se comparan los desplazamientos máximos de las pilas en dirección longitudinal (eje X) del puente, obtenidos del análisis de historias en el tiempo para los acelerogramas definidos anteriormente.

\section{FUERZA CORTANTE EN DIRECCIÓN LONGITUDINAL DEL PUENTE (EJE X) DE LA PILA 7 PARA EL SISMO DE PRETOLIA [KN]}

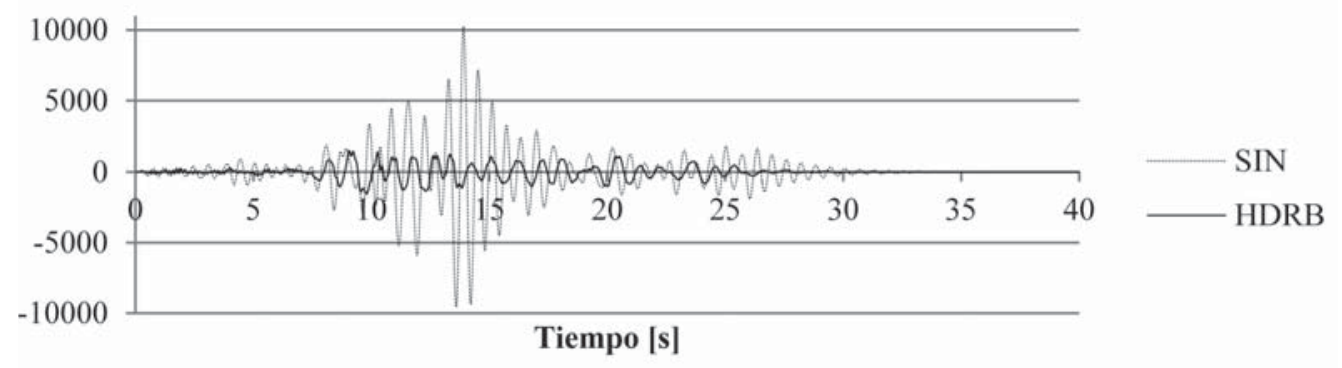

Figura 14. Fuerza cortante en la pila 7 en dirección longitudinal del puente para el sismo de Pretolia, sin aislamiento versus el aislamiento HDRB.

Fuente: elaboración propia 
Tabla 6. Comparación de los desplazamientos máximos de las pilas en dirección longitudinal (eje X) del puente para los modelos sin sistema de aislamiento, con HDRB, con FPS y con LRB

\begin{tabular}{|c|c|c|c|c|c|c|c|c|c|c|c|c|}
\hline \multirow[b]{3}{*}{ SISMO } & \multicolumn{12}{|c|}{ Comparación de los desplazamientos en X [mm] } \\
\hline & \multicolumn{4}{|c|}{ PILA 1} & \multicolumn{4}{|c|}{ PILA 2} & \multicolumn{4}{|c|}{ PILA 3} \\
\hline & SIN & LRB & HDRB & FPS & SIN & LRB & HDRB & FPS & $\mathrm{SIN}$ & LRB & HDRB & FPS \\
\hline COALIGNA & 69.5 & 15.5 & 15.9 & 6.4 & 47.3 & 33.0 & 30.9 & 28.6 & 44.0 & 44.4 & 35.4 & 41.1 \\
\hline DUZCE & 29.3 & 9.5 & 10.7 & 19.4 & 112.0 & 19.9 & 23.7 & 19.3 & 181.3 & 27.2 & 36.0 & 17.8 \\
\hline FRIULI & 8.5 & 4.2 & 6.5 & 9.6 & 15.2 & 6.0 & 8.3 & 7.6 & 9.4 & 9.0 & 11.9 & 7.2 \\
\hline HELENA & 58.3 & 16.3 & 14.3 & 11.8 & 51.5 & 39.3 & 38.0 & 24.5 & 45.5 & 53.4 & 52.0 & 38.4 \\
\hline LOMAPRIETA & 37.3 & 16.5 & 10.2 & 17.1 & 54.5 & 31.9 & 32.4 & 21.8 & 50.9 & 49.3 & 44.5 & 24.5 \\
\hline MAMMOTH & 29.3 & 14.0 & 7.7 & 9.2 & 33.2 & 20.3 & 19.6 & 23.9 & 24.1 & 24.8 & 25.5 & 25.0 \\
\hline MONTENEGRO & 54.7 & 18.3 & 11.1 & 16.3 & 57.4 & 25.6 & 25.3 & 37.8 & 45.6 & 35.4 & 32.4 & 35.5 \\
\hline PRETOLIA & 106.7 & 25.0 & 16.0 & 13.3 & 172.8 & 47.4 & 51.4 & 52.3 & 101.7 & 70.5 & 67.9 & 52.0 \\
\hline UMBRIA & 7.8 & 6.5 & 7.1 & 9.6 & 10.3 & 6.8 & 7.4 & 11.6 & 6.8 & 7.9 & 10.3 & 8.9 \\
\hline WHITTIER & 8.7 & 4.7 & 5.8 & 5.4 & 8.7 & 7.4 & 7.9 & 4.4 & 5.7 & 11.3 & 9.6 & 6.3 \\
\hline \multirow[t]{2}{*}{ Máximos } & 106.7 & 25.0 & 16.0 & 19.4 & 172.8 & 47.4 & 51.4 & 52.3 & 181.3 & 70.5 & 67.9 & 52.0 \\
\hline & \multicolumn{4}{|c|}{ PILA 4} & \multicolumn{4}{|c|}{ PILA 5} & \multicolumn{4}{|c|}{ PILA 6} \\
\hline SISMO & SIN & LRB & HDRB & FPS & SIN & LRB & HDRB & FPS & SIN & LRB & HDRB & FPS \\
\hline COALIGNA & 42.5 & 46.9 & 37.5 & 44.1 & 47.8 & 35.9 & 33.1 & 39.9 & 72.9 & 20.6 & 22.5 & 9.1 \\
\hline DUZCE & 180.4 & 29.4 & 38.3 & 18.9 & 161.7 & 22.6 & 29.2 & 17.6 & 28.2 & 12.2 & 15.9 & 17.4 \\
\hline FRIULI & 8.0 & 9.5 & 12.8 & 7.3 & 12.6 & 7.2 & 9.3 & 7.0 & 8.1 & 5.0 & 6.7 & 9.4 \\
\hline HELENA & 44.4 & 57.8 & 55.0 & 41.7 & 48.4 & 44.1 & 44.0 & 36.5 & 74.6 & 23.2 & 20.2 & 14.7 \\
\hline LOMAPRIETA & 48.4 & 52.3 & 46.1 & 25.2 & 55.8 & 38.4 & 38.3 & 24.3 & 45.2 & 19.7 & 16.2 & 25.6 \\
\hline МАММОТН & 23.3 & 24.2 & 26.5 & 25.3 & 27.0 & 22.7 & 22.8 & 24.3 & 26.9 & 16.5 & 10.2 & 15.2 \\
\hline MONTENEGRO & 46.4 & 37.7 & 33.4 & 37.0 & 53.0 & 29.3 & 31.1 & 36.0 & 37.2 & 22.1 & 16.3 & 22.2 \\
\hline PRETOLIA & 95.2 & 76.1 & 71.5 & 55.6 & 115.7 & 51.1 & 62.9 & 51.4 & 107.8 & 35.2 & 23.5 & 21.4 \\
\hline UMBRIA & 6.9 & 7.7 & 10.4 & 8.8 & 7.5 & 7.6 & 6.9 & 8.8 & 6.6 & 8.2 & 6.7 & 11.7 \\
\hline WHITTIER & 5.4 & 11.9 & 9.8 & 6.4 & 6.6 & 8.1 & 8.4 & 6.0 & 11.4 & 5.5 & 5.9 & 4.7 \\
\hline Máximos & 180.4 & 76.1 & 71.5 & 55.6 & 161.7 & 51.1 & 62.9 & 51.4 & 107.8 & 35.2 & 23.5 & 25.6 \\
\hline
\end{tabular}


reflexión

\begin{tabular}{|l|c|c|c|c|c|c|c|c|}
\hline & \multicolumn{4}{|c|}{ PILA 7 } & \multicolumn{4}{c|}{ PILA 8 } \\
\hline SISMO & SIN & LRB & HDRB & FPS & SIN & LRB & HDRB & FPS \\
\hline COALIGNA & 23.9 & 9.0 & 7.3 & 4.3 & 9.0 & 0.6 & 7.3 & 1.2 \\
\hline DUZCE & 24.5 & 6.6 & 4.2 & 5.8 & 17.1 & 0.8 & 0.7 & 1.5 \\
\hline FRIULI & 9.4 & 3.0 & 2.8 & 4.7 & 9.2 & 0.6 & 0.3 & 0.4 \\
\hline HELENA & 27.2 & 8.4 & 6.7 & 4.3 & 16.4 & 1.0 & 1.2 & 1.1 \\
\hline LOMAPRIETA & 24.3 & 8.0 & 6.0 & 4.7 & 33.0 & 1.0 & 0.9 & 0.9 \\
\hline MAMMOTH & 27.4 & 8.6 & 4.5 & 2.2 & 15.9 & 1.2 & 0.9 & 0.7 \\
\hline MONTENEGRO & 55.9 & 10.3 & 5.7 & 8.3 & 22.1 & 1.3 & 1.2 & 1.1 \\
\hline PRETOLIA & 68.2 & 10.7 & 6.8 & 4.1 & 23.0 & 1.7 & 1.9 & 1.6 \\
\hline UMBRIA & 16.1 & 4.4 & 3.4 & 4.2 & 20.2 & 0.7 & 0.4 & 0.5 \\
\hline WHITTIER & 5.0 & 2.1 & 5.2 & 5.3 & 11.5 & 0.4 & 0.5 & 0.5 \\
\hline Máximos & 68.2 & 10.7 & 7.3 & 8.3 & 33.0 & 1.7 & 7.3 & 1.6 \\
\hline
\end{tabular}

Fuente: elaboración propia

En la tabla 7 se comparan las fuerzas cortantes máximas para cada pila en dirección longitudinal (eje X) del puente, obtenidos del análisis de historias en el tiempo para los acelerogramas definidos anteriormente.

En la tabla 8 se comparan los desplazamientos y en la tabla 9 las fuerzas cortantes en dirección longitudinal del puente, para el modelo sin aislamiento sísmico, como también para los modelos con los tres sistemas de aislamiento de base, para los valores máximos obtenidos del análisis de historias en el tiempo, para los diez acelerogramas de la falla Frontal y la falla BucaramangaSantaMarta.

La mayor deriva del puente sin aislamiento sísmico en dirección longitudinal se presenta en la pila 2 y es del $0,45 \%$, y por el uso de aisladores de base, se reduce al orden del 0,14\%, también se puede observar que las derivas en dirección transversal del puente sin aislamiento sísmico son imperceptibles, mientras que con el uso de aisladores de base aumentan considerablemente, alcanzándose valores máximos de deriva del orden del $0,16 \%$. Aunque también se percibe que las pilas del puente sin aislamiento sísmico son altamente rígidas pues, en general, las derivas son muy pequeñas, valores desde $0,21 \%$ hasta $0,45 \%$.

Se hace evidente que, cuando se usa cualquiera de los tres sistemas de aislamiento sísmico, las fuerzas cortantes en dirección longitudinal del puente que actúan en las pilas, que son las más relevantes, se reducen de manera significativa; por ejemplo en la pila 7 de tener una fuerza cortante de $10246 \mathrm{KN}$ para el puente sin aislamiento, se puede llevar hasta un valor de $1705 \mathrm{KN}$ usando un sistema de aislamiento HDRB.

En la figura 15 se muestran los resultados de las tablas 8 y 9 , donde se comparan los desplazamientos y fuerzas cortantes en dirección longi- 
Tabla 7. Comparación de las fuerzas cortantes máximas para cada pila en dirección longitudinal (eje X) del puente para los modelos sin sistema de aislamiento, con HDRB, con FPS y con LRB

\begin{tabular}{|c|c|c|c|c|c|c|c|c|c|c|c|c|}
\hline \multirow[b]{3}{*}{ SISMO } & \multicolumn{12}{|c|}{ Comparación de las fuerzas cortantes en X [KN] } \\
\hline & \multicolumn{4}{|c|}{ PILA 1} & \multicolumn{4}{|c|}{ PILA 2} & \multicolumn{4}{|c|}{ PILA 3} \\
\hline & $\mathrm{SIN}$ & LRB & HDRB & FPS & SIN & LRB & HDRB & FPS & SIN & LRB & HDRB & FPS \\
\hline COALIGNA & 3906 & 1560 & 1560 & 713 & 1013 & 979 & 853 & 899 & 1200 & 829 & 566 & 887 \\
\hline DUZCE & 1569 & 948 & 1077 & 1949 & 1465 & 593 & 675 & 578 & 1359 & 961 & 577 & 398 \\
\hline FRIULI & 486 & 414 & 666 & 959 & 867 & 189 & 266 & 221 & 1119 & 475 & 311 & 1551 \\
\hline HELENA & 3258 & 1654 & 1329 & 1130 & 1258 & 1127 & 1060 & 748 & 1262 & 1142 & 940 & 825 \\
\hline LOMAPRIETA & 1969 & 1665 & 1225 & 1721 & 975 & 943 & 888 & 622 & 1119 & 949 & 882 & 542 \\
\hline МАMMOTH & 1568 & 1397 & 730 & 981 & 499 & 601 & 545 & 700 & 448 & 454 & 380 & 550 \\
\hline MONTENEGRO & 2922 & 1807 & 1143 & 1681 & 1256 & 756 & 690 & 1102 & 2354 & 715 & 639 & 729 \\
\hline PRETOLIA & 5876 & 2508 & 366 & 1320 & 1928 & 1344 & 1357 & 1618 & 1458 & 1054 & 1165 & 1177 \\
\hline UMBRIA & 408 & 640 & 627 & 962 & 954 & 208 & 230 & 333 & 1621 & 395 & 371 & 196 \\
\hline \multirow[t]{2}{*}{ WHITTIER } & 549 & 490 & 597 & 546 & 1719 & 220 & 256 & 1402 & 1081 & 368 & 469 & 106 \\
\hline & \multicolumn{4}{|c|}{ PILA 4} & \multicolumn{4}{|c|}{ PILA 5} & \multicolumn{4}{|c|}{ PILA 6} \\
\hline SISMO & $\mathrm{SIN}$ & LRB & HDRB & FPS & SIN & LRB & HDRB & FPS & SIN & LRB & HDRB & FPS \\
\hline COALIGNA & 894 & 893 & 551 & 862 & 1507 & 830 & 724 & 900 & 2604 & 1445 & 1495 & 674 \\
\hline DUZCE & 1346 & 814 & 571 & 372 & 1405 & 523 & 619 & 423 & 914 & 873 & 1071 & 1139 \\
\hline FRIULI & 1149 & 402 & 270 & 119 & 1131 & 189 & 261 & 164 & 497 & 356 & 458 & 633 \\
\hline HELENA & 1298 & 1186 & 932 & 834 & 1265 & 914 & 934 & 844 & 2386 & 1622 & 1355 & 953 \\
\hline LOMAPRIETA & 1296 & 947 & 894 & 422 & 1084 & 864 & 783 & 561 & 1559 & 1350 & 1103 & 1694 \\
\hline MAMMOTH & 425 & 432 & 364 & 484 & 470 & 530 & 477 & 536 & 908 & 1156 & 696 & 1016 \\
\hline MONTENEGRO & 2208 & 672 & 606 & 676 & 2294 & 702 & 603 & 763 & 1153 & 1503 & 1094 & 1101 \\
\hline PRETOLIA & 1311 & 1117 & 1152 & 1092 & 1681 & 1074 & 1192 & 1224 & 3466 & 238 & 1542 & 1488 \\
\hline UMBRIA & 1703 & 358 & 338 & 152 & 1298 & 185 & 216 & 219 & 353 & 563 & 462 & 770 \\
\hline WHITTIER & 1092 & 330 & 378 & 203 & 1248 & 200 & 281 & 132 & 658 & 387 & 415 & 358 \\
\hline Máximos & 2208 & 1186 & 1152 & 1092 & 2294 & 1074 & 1192 & 1224 & 3466 & 1622 & 1542 & 1694 \\
\hline
\end{tabular}


reflexión

\begin{tabular}{|l|c|c|c|c|c|c|c|c|}
\hline & \multicolumn{4}{|c|}{ PILA 7 } & \multicolumn{4}{c|}{ PILA 8 } \\
\hline SISMO & SIN & LRB & HDRB & FPS & SIN & LRB & HDRB & FPS \\
\hline COALIGNA & 3596 & 2150 & 1705 & 999 & 2630 & 260 & 683 & 357 \\
\hline DUZCE & 3682 & 1546 & 983 & 1247 & 5160 & 329 & 279 & 517 \\
\hline FRIULI & 1409 & 702 & 597 & 1019 & 2598 & 253 & 108 & 109 \\
\hline HELENA & 4086 & 1981 & 1588 & 912 & 4830 & 444 & 531 & 335 \\
\hline LOMAPRIETA & 3648 & 1897 & 1381 & 1100 & 9720 & 467 & 346 & 297 \\
\hline MAMMOTH & 4114 & 2008 & 1019 & 588 & 4646 & 533 & 372 & 990 \\
\hline MONTENEGRO & 8398 & 2366 & 1324 & 1871 & 6500 & 568 & 446 & 255 \\
\hline PRETOLIA & 10246 & 2528 & 1549 & 949 & 6778 & 719 & 746 & 539 \\
\hline UMBRIA & 2392 & 1038 & 763 & 900 & 5944 & 286 & 138 & 1140 \\
\hline WHITTIER & 736 & 465 & 1179 & 1187 & 3104 & 119 & 119 & 98 \\
\hline Máximos & 10246 & 2528 & 1705 & 1871 & 9720 & 719 & 746 & 1140 \\
\hline
\end{tabular}

Fuente: elaboración propia
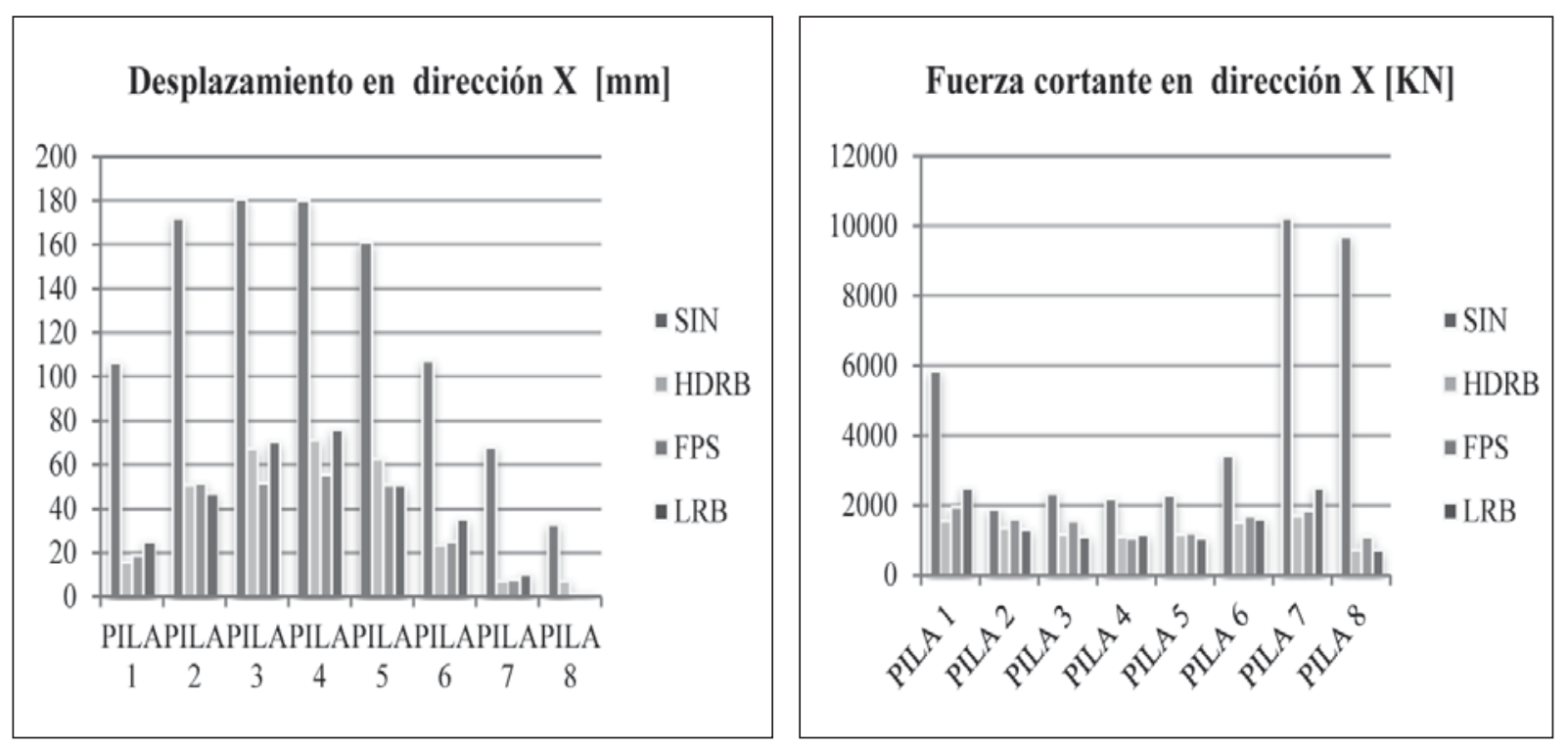

Figura 15. Comparación de desplazamientos y fuerzas cortantes en dirección longitudinal y transversal del puente con y sin aislamiento de base.

Fuente: elaboración propia 
Tabla 8. Comparación de desplazamientos para los cuatro modelos del puente, en dirección longitudinal y transversal del puente.

\begin{tabular}{|c|c|c|c|c|c|c|c|c|c|c|}
\hline & & & PILA 1 & PILA 2 & PILA 3 & PILA 4 & PILA 5 & PILA 6 & PILA 7 & PILA 8 \\
\hline \multirow{8}{*}{$\begin{array}{l}\text { Desplazamientos } \\
\text { y derivas en } X\end{array}$} & \multirow{2}{*}{ SIN } & {$[\mathrm{mm}]$} & 106.70 & 172.80 & 181.30 & 180.40 & 161.70 & 107.80 & 68.19 & 33.00 \\
\hline & & & $0.43 \%$ & $0.45 \%$ & $0.40 \%$ & $0.39 \%$ & $0.37 \%$ & $0.38 \%$ & $0.36 \%$ & $0.21 \%$ \\
\hline & \multirow{2}{*}{ HDRB } & {$[\mathrm{mm}]$} & 15.99 & 51.40 & 67.93 & 71.51 & 62.90 & 23.46 & 7.33 & 7.28 \\
\hline & & & $0.06 \%$ & $0.13 \%$ & $0.15 \%$ & $0.16 \%$ & $0.14 \%$ & $0.08 \%$ & $0.04 \%$ & $0.05 \%$ \\
\hline & \multirow{2}{*}{ FPS } & {$[\mathrm{mm}]$} & 19.42 & 52.32 & 51.98 & 55.60 & 51.44 & 25.58 & 8.29 & 1.59 \\
\hline & & & $0.08 \%$ & $0.14 \%$ & $0.12 \%$ & $0.12 \%$ & $0.12 \%$ & $0.09 \%$ & $0.04 \%$ & $0.01 \%$ \\
\hline & \multirow{2}{*}{ LRB } & {$[\mathrm{mm}]$} & 25.04 & 47.41 & 70.45 & 76.13 & 51.14 & 35.24 & 10.67 & 1.69 \\
\hline & & & $0.10 \%$ & $0.12 \%$ & $0.16 \%$ & $0.17 \%$ & $0.12 \%$ & $0.12 \%$ & $0.06 \%$ & $0.01 \%$ \\
\hline
\end{tabular}

Fuente: elaboración propia

Tabla 9. Comparación de fuerzas cortantes para los cuatro modelos del puente, en dirección longitudinal y transversal del puente.

\begin{tabular}{|c|c|c|c|c|c|c|c|c|c|}
\hline \multicolumn{2}{|c}{} & PILA 1 & PILA 2 & PILA 3 & PILA 4 & PILA 5 & PILA 6 & PILA 7 & PILA 8 \\
\hline \multirow{4}{*}{ Fuerza cortante en X [KN] } & SIN & 5876 & 1928 & 2354 & 2208 & 2294 & 3466 & 10246 & 9720 \\
\cline { 2 - 11 } & HDRB & 1560 & 1357 & 1165 & 1152 & 1192 & 1542 & 1705 & 745.6 \\
\cline { 2 - 11 } & FPS & 1949 & 1618 & 1551 & 1092 & 1224 & 1694 & 1871 & 1140 \\
\cline { 2 - 11 } & LRB & 2508 & 1344 & 1142 & 1186 & 1074 & 1622 & 2528 & 718.6 \\
\hline
\end{tabular}

Fuente: elaboración propia

tudinal y transversal del puente con y sin aislamiento de base.

\section{CONCLUSIONES}

El uso de aisladores modificó la respuesta dinámica del puente en cuanto a los modos de vibración ya que, para el puente sin aislamiento, los modos principales son longitudinales, mientras que para el puente con sistemas de aislamiento el primer modo está en dirección longitudinal y el segundo modo de vibración está en dirección transversal, y para los modos superiores se repite este comportamiento.

En cuanto a los desplazamientos y fuerzas cortantes en el puente sin aisladores, son relevantes sólo en la dirección longitudinal de éste; los desplazamientos son máximos en las pilas centrales, que son las más altas, mientras que las fuerzas cortantes son muy altas en las pilas más cortas; y con el uso de sistemas de aislamiento de base, este comportamiento cambia totalmente: primero, las fuerzas cortantes y desplazamientos se hacen relevantes en las dos direcciones; segundo, las fuer- 
zas cortantes muy altas ya no se localizan en las pilas cortas, si no que tienden a ser uniformes en todas las pilas para las dos direcciones del puente; y en cuanto a los desplazamientos, siguen siendo mayores en las pilas altas, pero son de magnitudes muy parecidas en las dos direcciones.

Respecto a los tres sistemas de aislamiento de base usados, el HDRB, el LRB y el FPS, se puede ver que inducen un comportamiento muy similar en el puente, dando una respuesta parecida en fuerzas cortantes y desplazamientos sobre las pilas. Aunque si se analizan los desplazamientos que producen cada uno de los sistemas de aislamiento, se ve que el FPS, en general, es el que produce los más bajos; pero si se analizan las fuerzas cortantes en las pilas, el aislador HDRB es el que produce las menores.

\section{REFERENCIAS}

[1] D. Jara y R. J Casas, "Criterios de diseño sísmico de puentes", en Enfoques en la investigación científica: Producción actual en las universidades de Barcelona, [actas del VII Seminario de la APEC], Barcelona, 17 de mayo de 2002, pp. 127 - 136.

[2] A. Ghobarah, and H. M. Ali, "Seismic Performance of Highway Bridges", Engineering Structures, Vol. 10, No. 3, pp.157-166, 1988.

[3] D. H. Turkington, A. J. Carr, N.Cooke and P. J. Moss, "Seismic Design of Bridges on Lead-Rubber Bearings", Journal of Structural Engineering, ASCE, No.115, pp.3000-3016.

[4] J. S. Hwang and L.H. Sheng, "Equivalent Elastic Seismic Analysis of Base-Isolated Bridges with Lead-Rubber Bearings", Engineering Structures, Vol. 16, No. 3, pp.201-209.

[5] A. Kartoum, M. C. Constantinou and A. M. Reinhorn, "Sliding Isolation Seismic Response of Simply Supported Base-Isolated Bridge with Different Isolators" Int. J. Appl. Sci. Eng., 2006. 4, 1 69, System for Bridges: Analytical Study. Earthquake Spectra, Vol. 8, No. 3, pp. 345-372.
[6] Y. P. Wang, L. L Chung and W. H. Liao, "Seismic Response Analysis of Bridges Isolated with Friction Pendulum Bearings", Earthquake Engineering and Structural Dynamics, Vol. 27, No. 10, pp.1069-1093.

[7] R. S. Jangid, "Seismic Response of Isolated Bridges", Journal of Bridge Engineering, ASCE, Vol. 9, No. 2, pp.156-166.

[8] M. C. Kunde and R. S. Jangid, "Seismic Behavior of Isolated Bridges: A State-ofthe-Art Review", Electronic Journal of Structural Engineering, No. 3, pp.140170.

[9] J. C. Simo and J. M. Kelly, "The Analysis of Multilayer Elastomeric Bearings", Journal of Applied Mechanics, ASME, Vol. 51, No. 2, pp. 244-250, 1978

[10] F. Naeim and J.M. Kelly, Design of Seismic Isolated Structures: From Theory to Practice, New York: John Wiley \& Sons Inc., 1999.

[11] R. I. Skinner, J. M. Kelly and A. J. Heine, "Hysteretic Dampers for Earthquake-Resistant Structures", Earthquake Engineering and Structural Dynamics, Vol. 3, No. 3, pp. 287-296, 1975. 
[12] W. H. Robinson, "Lead-Rubber Hysteretic Bearings Suitable for Protecting Structures During Earthquakes", Earthquake Engineering and Structural Dynamics, Vol. 10, No. 4, pp.593-604, 1982.

[13] V. A. Zayas, S.S. Low and S.A. Mahin, "A simple pendulum technique for achieving seismic isolation", Earthquake Spectra, Vol. 6, pp.317-334,1990.

[14] Federal Emergency Management Agency (FEMA), NEHRP Guidelines for the Seismic Rehabilitation of Buildings and NEHER Commentary on the Guidelines for the Seismic Rehabilitation of Buildings, [Reports No. FEMA-273 and FEMA 274], Building Seismic Safety Council, Washington, DC, 1997.
[15] M. C. Constantinou, P. Tsopelas, A. Kasalanati and E. Wolff, Property Modification Factors for Seismic Isolation Bearings, [Report no. NCEER-99-0012], Multidisciplinary Center for Earthquake Engineering Research, Buffalo, New York, 1999.

[16] INGEOMINAS, Zonificación Sismogeotécnica indicativa del área metropolitana de Bucaramanga, 2001

[17] R. A. Ortíz y J. F. Gélvez, Espectros Generalizados de Deriva para Bucaramanga Usando un Modelo Continuo, [Trabajo de Grado], Universidad Industrial de Santander, Colombia, 2008 